\title{
A case report: delayed high fever and maculopapules during Sorafenib treatment of ectopic hepatocellular carcinoma
}

\author{
Tianxiang Cui ${ }^{1}$, Xinwei Diao ${ }^{2}$, Xiewan Chen ${ }^{3}$, Shaojiang Huang ${ }^{1}$ and Jianguo Sun ${ }^{1 *}$
}

\begin{abstract}
Background: Sorafenib is the standard first-line therapy for hepatocellular carcinoma (HCC) and probably ectopic hepatocellular carcinoma (EHCC) as well. No report involves a side effect of delayed high fever of sorafenib. This manuscript describes a case of EHCC in the thoracic and abdominal cavities, who showed a delayed high fever and maculopapules during sorafenib treatment.

Case presentation: The patient is a 63-year-old Chinese male with advanced EHCC, taking sorafenib 400 mg twice daily. On the tenth day, red maculopapules appeared all over the body. On the same day, the patient began to suffer from continuous high fever. Due to these effects, the patient was asked to cease sorafenib treatment, and the high fever and maculopapules were alleviated quickly. However, the symptoms were present again upon re-challenge of sorafenib. Prednisone was then administered to control the symptoms, with the dosage gradually reduced from 30 to $5 \mathrm{mg} /$ day in 1.5 months. No recurrence of fever or maculopapules has been found. Tumor response reached partial response (PR) and progression free survival (PFS) reached 392 days + by the date of Apr. 14th, 2016.
\end{abstract}

Conclusion: EHCC could be treated like orthotopic HCC by oral administration of sorafenib, which shows good tumor response and survival benefit. Delayed high fever and maculopapules are potential, rare and severe side effects of sorafenib, and could be effectively controlled by glucocorticoid.

Keywords: Sorafenib, Delayed reaction, High fever, Maculopapules, Ectopic hepatocellular carcinoma

\section{Background}

Ectopic hepatocellular carcinoma (EHCC) is a rare malignancy from ectopic liver, a kind of developmental abnormality [1-3]. The incidence of ectopic liver is only about $0.27-0.7 \%$ [3-5]. However, ectopic liver is more likely to develop primary hepatocellular carcinoma (HCC) compared with normal liver tissues. Although sorafenib is the standard first-line therapy for HCC, there is rare report on the role of sorafenib in treating EHCC [2]. Here we report a case of EHCC in the thoracic and abdominal cavities, who interestingly showed symptoms of delayed high fever and maculopapules during sorafenib treatment.

\footnotetext{
* Correspondence: sunjg09@aliyun.com

${ }^{1}$ Cancer Institute of PLA, Xinqiao Hospital, Third Military Medical University, Chongqing 400037, China

Full list of author information is available at the end of the article
}

\section{Case presentation}

A 63-year-old male presented with a history of hepatitis B virus (HBV) infection for 20 years and no anti-HBV treatment. In mid-Jan. 2015, the patient developed progressive and dull chest pain without obvious inducement. Chest CT scan revealed multiple pulmonary nodules and enlarged mediastinal lymph nodes. The level of serum alpha-fetal protein (AFP) reached $24793 \mathrm{ng} / \mathrm{mL}$. Due to no hepatic lesion found by contrast abdominal CT and MRI scan, F-18 positron emission tomography-computed tomography (PET/CT) was carried out. Unexpectedly, PET/CT definitely showed that multiple lesions in the thoracic and abdominal cavities, including several lung nodules in the maximum size of $1.7 \mathrm{~cm} \times 1.7 \mathrm{~cm}$ (Fig. 1 a1), mediastinal lymph nodes in the maximum size of $6.4 \mathrm{~cm} \times 3.2 \mathrm{~cm}$ (Fig. $1 \mathrm{a} 2$ ), and intraperitoneal mass in the fundus of stomach, cardia, portal fissure and abdominal aortas in the size of $4.6 \mathrm{~cm} \times 2.2 \mathrm{~cm}$ (Fig. $1 \mathrm{a}$ ), but still no hepatic lesion (Fig. 2a-d). On Mar. 6th, 2015, the patient underwent a 


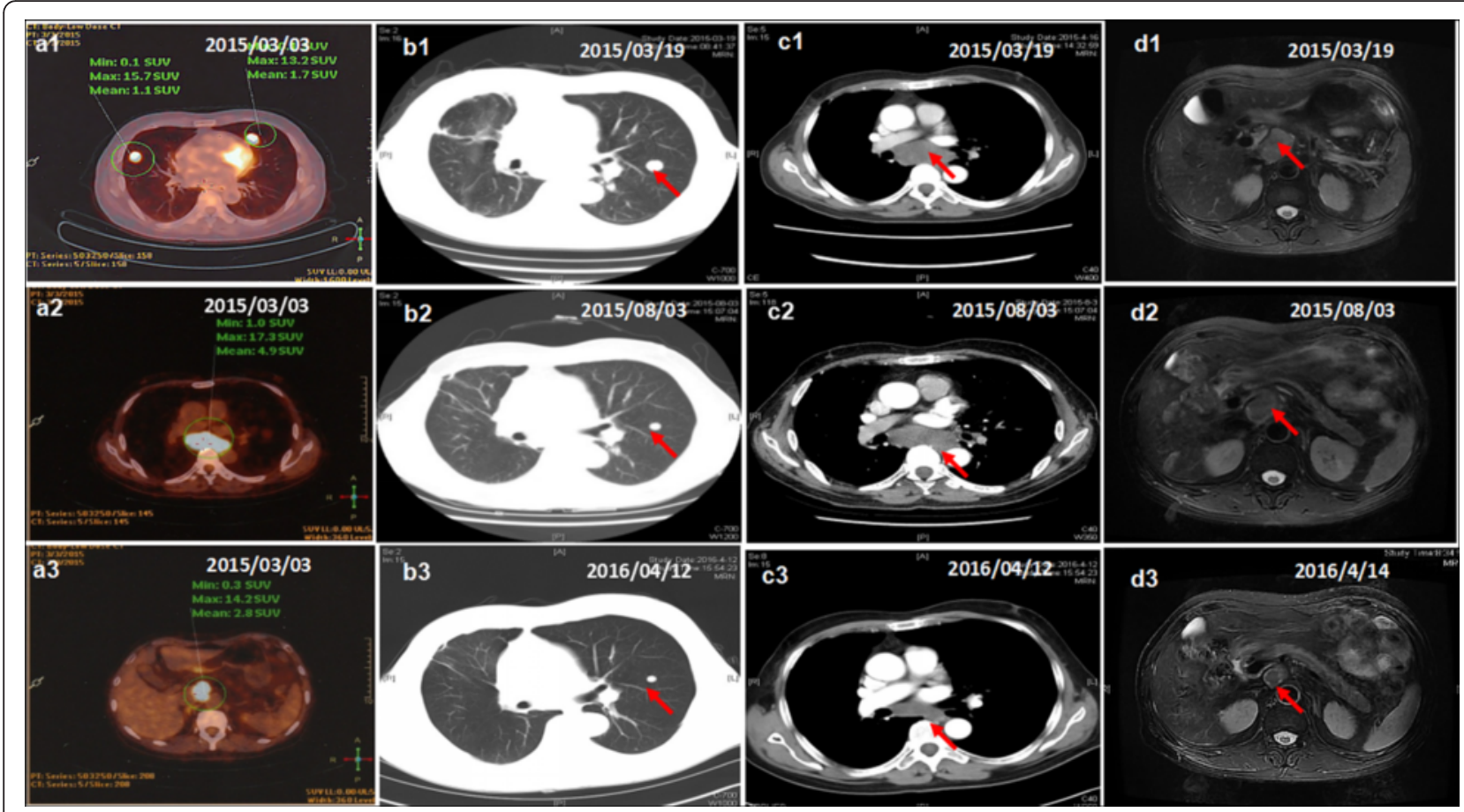

Fig. 1 Tumor response during sorafenib treatment. PET/CT showed multiple lesions in both lungs (a1), mediastinal lymph nodes (a2) and abdominal cavity (a3). No lesion was found in the liver by PET/CT (a3), abdominal MRI (d1-d3). The tumor response in both intrathoracic and intraperitoneal lesions dramatically shrank (b1-b3, c1-c3 and d1-d3)

wedge resection of right pulmonary. The pathological morphology displayed cancer cell nest with pseudoglandular structure and focal necrosis area, typical hepatocellular carcinoma cells with polygonal shape, eosinophilic cytoplasm and big anachromasis nucleus. And immunohistochemistry showed the expressions of CK18 ++, Glypican-3 ++ , Hepatocyte +, P53 + and Ki-67 $60-70 \%$, which confirmed the diagnosis of primary HCC (Fig. $3 \mathrm{a}-\mathrm{f}$ ). Thus, the patient was a rare case of EHCC in an advanced stage.
Following the standard therapy of HCC in National Comprehensive Cancer Network (NCCN) guidelines [6], the patient began to take sorafenib $400 \mathrm{mg}$ twice daily together with Chinese medicines (Jinlong capsule, Jiansheng Company, China) and immunopotentiative agents (ubenimex capsules, Yuandong Company, China) on Mar. 19th, 2015. On the tenth day (Mar. 28th, 2015), red maculopapules appeared in the face, neck, abdomen, back and legs (Fig. $4 \mathrm{a}$ and b). From the same day, the

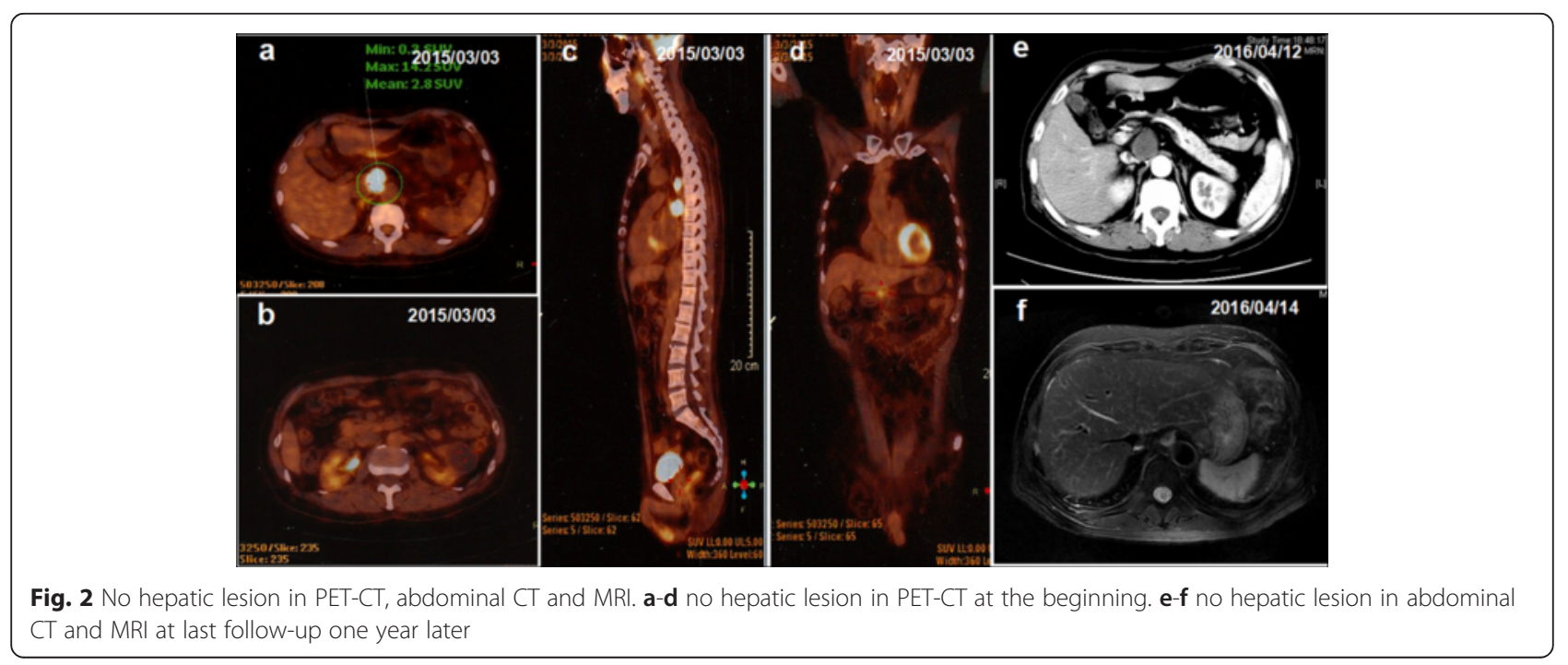




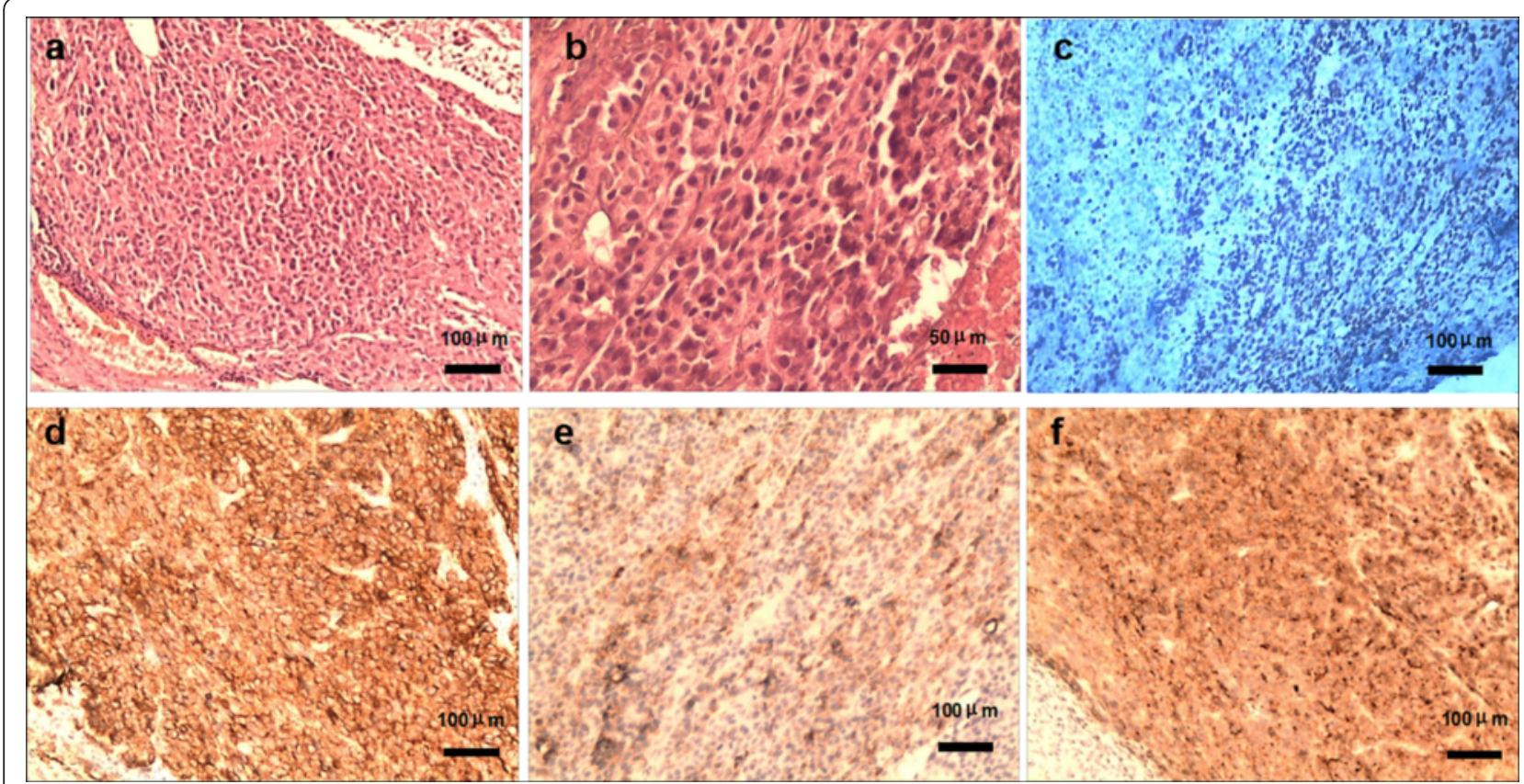

Fig. 3 Pathological diagnosis of ectopic hepatocellular carcinoma. a HE staining, cancer cell nest with pseudoglandular structure and focal necrosis area $(100 \times)$. b HE staining, morphologically typical hepatocellular carcinoma cells with polygonal shape, eosinophilic cytoplasm and big anachromasis nucleus. c Ki-67, positive in nucleus (60-70 \%, 100X). d CK18, positive on membrane $(++, 100 \times)$. e Hepatocyte, positive in cytoplasm $(+, 100 \times) . \mathbf{f}$ Glypican-3, positive in cytoplasm $(++, 100 x)$

patient began to suffer from continuous high fever, with highest body temperature ranging from $39.0^{\circ} \mathrm{C}$ to $39.6^{\circ}$ $\mathrm{C}$ each day. The patient did not decrease the dose or stop the use of sorafenib despite feeling dizzy and fatigue. There was no abnormality in blood routine or blood culture examination, no pulmonary infection or other inflammatory signs. After taking non-steroidal drugs with antipyretic and anti-allergic properties and withdrawing all drugs except sorafenib, no significant improvement was observed in high fever and maculopapules. Therefore, the patient was asked to cease the use of sorafenib from Apr. 3rd, 2015. Strikingly, the high fever and maculopapules were alleviated quickly. On the third day, the body temperature returned to the normal level, and on the fourth day, the maculopapules almost completely disappeared. Next, the patient began to take sorafenib again at the standard dose of $400 \mathrm{mg}$ twice daily on Apr. 7th, 2015. As expected, the fever

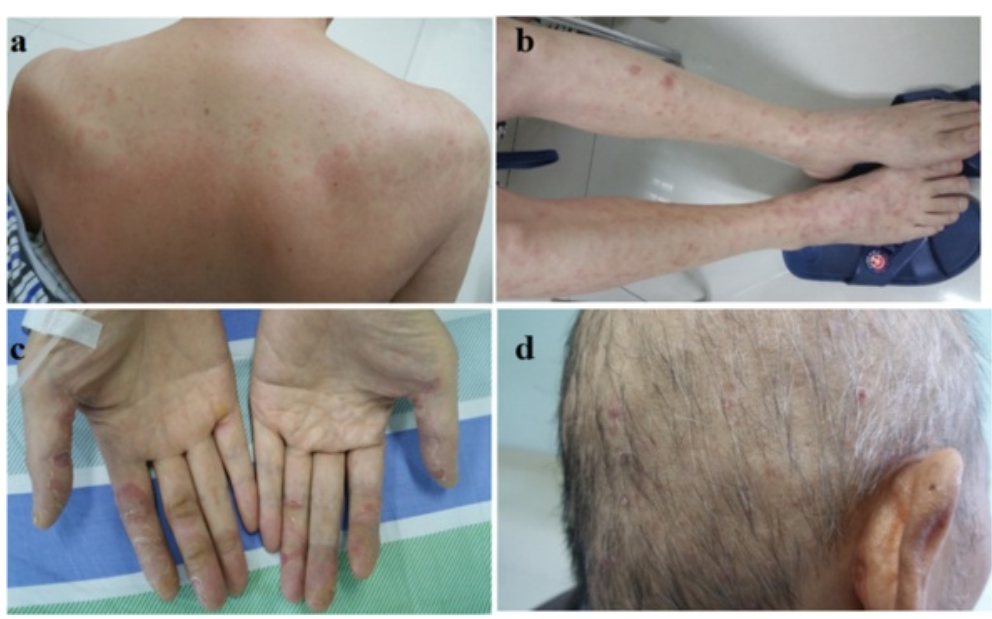

Fig. 4 Skin reaction all over the body. Red maculopapules in the back and legs (a, b). Hand-foot skin reaction in both hands (c). Rash in the scalp and ears (d) 
developed, raising the body temperature to $38.1{ }^{\circ} \mathrm{C}$ on exactly the same day, to $38.4{ }^{\circ} \mathrm{C}$ the next day and $38.8^{\circ} \mathrm{C}$ the third day. Also, the red maculopapules relapsed at the same time. Hence, prednisone was administered at a dosage of $30 \mathrm{mg} /$ day on the fourth day. The body temperature was decreased and maculopapules relieved quickly. The dosage of prednisone was gradually reduced from 30 to $10 \mathrm{mg} /$ day in one month, and then kept at $5 \mathrm{mg} /$ day for another two weeks. After the patient's temperature has returned to normal for more than one month, he intermittently took ubenimex and Jinlong capsule again. There has been no recurrence of fever and maculopapule to date. The dynamic change in body temperature is reflected in Fig. 5a. Other common side effects also need to be mentioned, such as hand-foot syndrome and rash in the scalp and ears and body (Fig. 4 c and d). During the follow-up, the tumor response of both intrathoracic and intraperitoneal lesions reached partial response (PR) according to RECIST 1.1 criteria. The lung nodules, mediastinal lymph nodes and intraperitoneal mass shrank gradually from Mar. 19, 2015 to Apr. 12th, 2016 (Fig. 1 b1-b3, c1-c3 and d1-d3), with the maximum size of $0.8 \mathrm{~cm} \times 0.6 \mathrm{~cm}, 3.7 \mathrm{~cm} \times 2.2 \mathrm{~cm}$ and $2.0 \mathrm{~cm} \times 1.9 \mathrm{~cm}$ at the date of Apr. 12th, 2016, respectively. There was still no lesion in liver, revealed by repeated examinations of abdominal ultrasound, CT and MRI (Fig. 2 e and f). In addition, serum AFP gradually dropped from $24793 \mathrm{ng} / \mathrm{mL}$ to $2.19 \mathrm{ng} / \mathrm{mL}$ on Apr. 12th, 2016 following the disease control (Fig. 5b). Until Apr. 14th, 2016 in the last review, the patient had maintained a good condition with progression free survival (PFS) of 392 days + .

\section{Conclusions}

Consistent with most $\mathrm{HCC}$ in orthotopic liver tissue, EHCC also arises as a result of chronic hepatitis B or C infection and secondary cirrhosis $[7,8]$. However, many factors including liver metabolism disorders, damage of cellular repair, and carcinogen microenvironment, cause a greatly higher incidence of EHCC than that of orthotopic HCC $[1,4,7]$. The diagnosis of EHCC mainly depends on surgery and pathologic diagnosis, and cannot be based on serum AFP level and medical history of hepatitis B virus infection. We report a classical case of EHCC here. First, this patient suffered from a 20-year HBV infection and mild hepatocirrhosis. Second, imaging techniques showed no tumor in the liver at initial diagnosis and during the thirteen-month follow-up. Third, postoperative serum AFP level was more than $16000 \mathrm{ng} / \mathrm{ml}$ and dropped dramatically following the disease control after sorafenib treatment. Last and most important, pulmonary lobectomy provided the pathologic diagnosis of HCC.

Sorafenib is a multi-kinase inhibitor that blocks signaling pathways in tumor growth $[9,10]$. In a global clinical trial of patients with advanced HCC in 2008, the median overall survival (mOS) in sorafenib group was 2.8 months longer than that in placebo group (10.7 months vs 7.9 months), and the mPFS in sorafenib group was 2.3 months longer than that in placebo group (5.5 months vs 2.8 months) [11]. And a phase III, randomised, double-blind, placebo-controlled trial in AsiaPacific region in 2009 revealed that mOS was 2.3 months longer in sorafenib group than in placebo group (6.5 months vs 4.2 months) [12]. In the current case, the

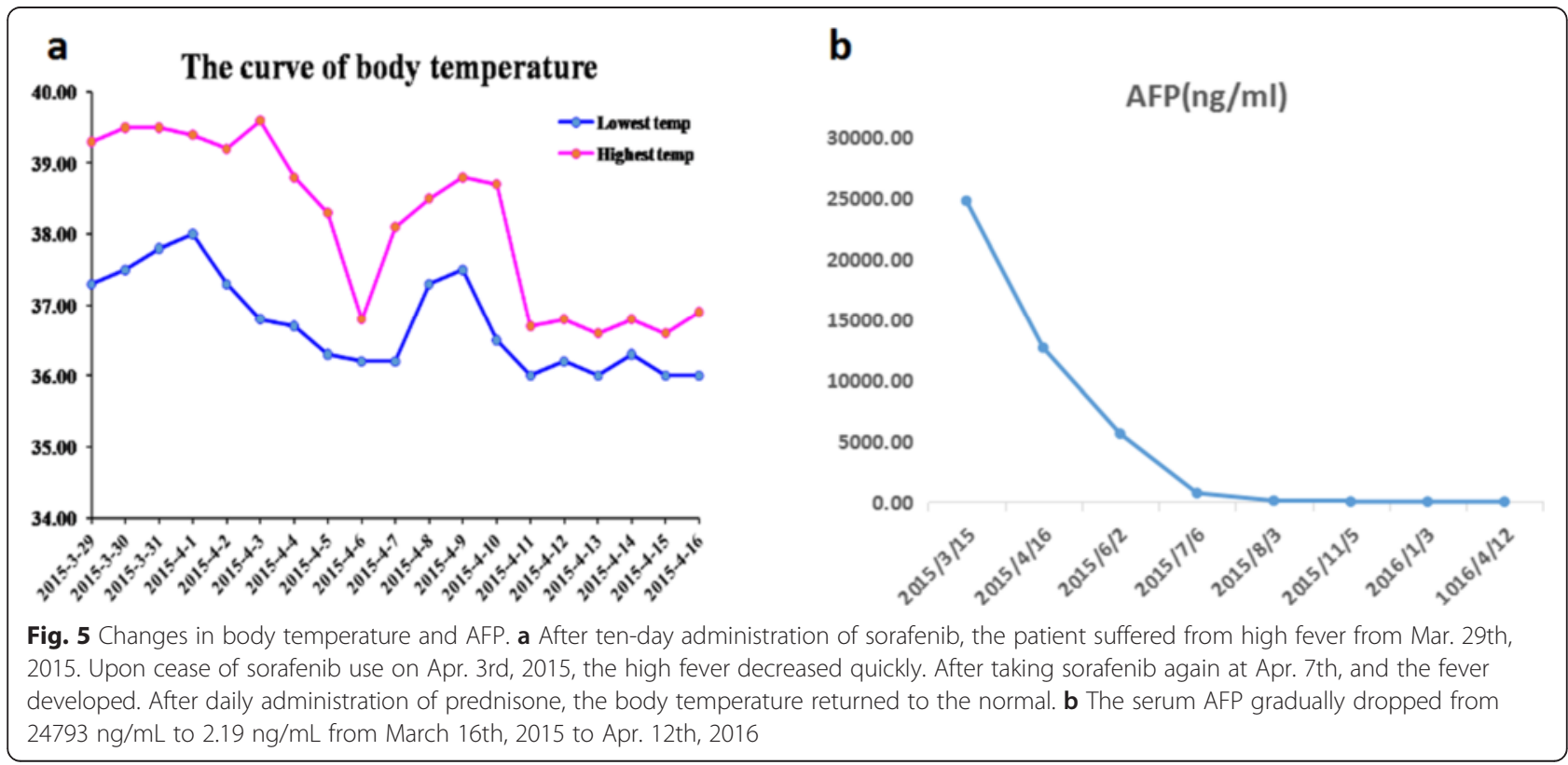


patient keeps a good condition with PFS of 392 days+, which is much longer than that in previous trials $[11,12]$. To the best of our knowledge, this is the first case report that describes sorafenib treatment in a case of EHCC.

Sorafenib also produces adverse effects including skin toxicity, gastrointestinal reaction, systemic reaction and vascular dysfunction, hoarseness, fever, pain, ulcers in the mouth, etc. Hand-foot syndrome and rash are the most common adverse events. In the Sorafenib Hepatocellular Carcinoma Assessment Randomized Protocol (SHARP) trial, hand-foot skin reaction occurred in up to $21 \%$ of enrolled patients [13]. Sorafenib treatment in HCC possibly causes rare side effects, such as rhabdomyolysis thyroid crisis and liver failure [14-17]. Most side effects could be alleviated after reducing or ceasing the use of the drug. To the best of our knowledge, this is the first case report that describes delayed high fever and maculopapules during sorafenib treatment as well. High fever and maculopapules rapidly relieved when ceasing sorafenib treatment and returned soon after sorafenib re-challenging. We propose that the delayed high fever and maculopapules are potential side effects of sorafenib. Additionally, we can exclude the cause of delayed high fever in the use of ubenimex and Jinlong capsule, because we stopped these medicines once the patient suffered from fever. However, there was no change in high fever. And when the patient took these medicine later, there has been no recurrence of fever.

In conclusion, EHCC could be treated like orthotopic HCC by orally administering sorafenib, and the treatment can achieve good tumor response and survival benefit. Delayed high fever and maculopapules are potential, rare and severe side effects of sorafenib, which could be effectively controlled by glucocorticoid.

\section{Abbreviations}

AFP, alpha-fetal protein; EHCC, ectopic hepatocellular carcinoma; HBV, hepatitis B virus; HCC, hepatocellular carcinoma; NCCN, National Comprehensive Cancer Network; PET/CT, positron emission tomography-computed tomography; PFS, progression free survival

\section{Acknowledgements}

The authors do not have any acknowledgements.

\section{Funding}

No funding was received.

\section{Availability of data and materials}

All data presented in the manuscript.

\section{Authors' contributions}

TC and SH participated in the treatment of this case. TC and JS drafted the manuscript. XD participated in the pathological diagnosis. XC aided with manuscript writing and language prettification. JS participated in the conception and design. All authors read and approved the final manuscript.

Competing interests

The authors declare that they have no competing interests.

\section{Consent for publication}

Written informed consent was obtained from the patient for publication of this case report and any accompanying images. A copy of the written consent is available for review by the Editor of this journal.

\section{Ethics approval and consent to participate}

This report has been approved by the Ethics Committee in our hospital. Consent to participate in this study was obtained from the patient.

\section{Author details}

${ }^{1}$ Cancer Institute of PLA, Xinqiao Hospital, Third Military Medical University, Chongqing 400037, China. ${ }^{2}$ Department of pathology, Xinqiao Hospital, Third Military Medical University, Chongqing 400037, China. ${ }^{3}$ Medical English Department, College of Basic Medicine, Third Military Medical University, Chongqing 400038, China.

Received: 27 January 2016 Accepted: 20 July 2016

Published online: 27 July 2016

\section{References}

1. Yamashita M, Nagamine Y, Ozaki K, Ueshima S, Takahashi H, Inoue H, et al. An autopsy case of a cirrhotic ectopic liver with a review of the literature. Acta Hepatol Jpn. 1985;26:510-4.

2. Pavel Z, Lubomir M, Peter I, Jan F. Ectopic liver: different manifestations, one solution. World J Gastroenterol. 2013;19(38):6485-9.

3. Arakawa M, Kimura Y, Sakata K, Kubo Y, Fukushima T, Okuda K. Propensity of ectopic liver to hepatocarcinogenesis: case reports and a review of the literature. Hepatology. 1999;29(1):57-61.

4. Watanabe M, Matsura T, Takatori Y, Ueki K, Kobatake T, Hidaka M, et al. Five cases of ectopic liver and a case of accessory lobe of the liver. Endoscopy. 1989;21(1):39-42.

5. Sato S, Watanabe M, Nagasawa S, Niigaki M, Sakai S, Akagi S. Laparoscopic observations of congenital anomalies of the liver. Gastrointest Endosc. 1998 ; 47(2):136-40.

6. Cainap C, Qin S, Huang WT, Chung IJ, Pan H, Cheng Y, et al. Linifanib versus Sorafenib in patients with advanced hepatocellular carcinoma: results of a randomized phase III trial. J Clin Oncol. 2015:33(2):172-9.

7. Caygill CP, Gatenby PA. Ectopic liver and hepatocarcinogenesis. Eur J Gastroenterol Hepatol. 2004;16(8):727-9.

8. Asselah T, Condat B, Cazals-Hatem D, Hassani Z, Bernuau J, Groussard O, et al. Ectopic hepatocellular carcinoma arising in the left chest wall: a longterm follow-up. Eur J Gastroenterol Hepatol. 2001;13(7):873-5.

9. Wilhelm SM, Carter C, Tang L, Wilkie D, McNabola A, Rong H, et al. BAY 439006 exhibits broad spectrum oral antitumor activity and targets the RAF/ MEK/ERK pathway and receptor tyrosine kinases involved in tumor progression and angiogenesis. Cancer Res. 2004;64(19):7099-109.

10. Carlomagno F, Anaganti S, Guida T, Salvatore G, Troncone G, Wilhelm SM, et al. BAY 43-9006 inhibition of oncogenic RET mutants. J Natl Cancer Inst. 2006;98(5):326-34

11. Llovet JM, Ricci S, Mazzaferro V, Hilgard P, Gane E, Blanc JF, et al. SHARP Investigators Study Group. Sorafenib in advanced hepatocellular carcinoma. N Engl J Med. 2008;359(4):378-90.

12. Cheng AL, Kang YK, Chen Z, Tsao CJ, Qin S, Kim JS, et al. Efficacy and safety of sorafenib in patients in the Asia-Pacific region with advanced hepatocellular carcinoma: a phase III randomised, double-blind, placebocontrolled trial. Lancet Oncol. 2009;10(1):25-34.

13. Rimassa L, Santoro A. Sorafenib therapy in advanced hepatocellular carcinoma: the SHARP trial. Expert Rev Anticancer Ther. 2009;9(6):739-45.

14. Tsuji K, Takemura K, Minami K, Teramoto R, Nakashima K, Yamada S, et al. A case of rhabdomyolysis related to sorafenib treatment for advanced hepatocellular carcinom. Clin J Gastrornterol. 2013;6(3):255-7.

15. Haraldsdottir S, Li Q, Villalona-Calero MA, Olencki TE, Kendra K, Ing SW. Case of sorafenib-induced thyroid storm. J Clin Oncol. 2013;31(16):262-4.

16. Hootegem AV, Verslype C, Van Steenbergen WV. Sorafenib-induced liver failure: a case report and review of the literature. Case Reports Hepatol. 2011;2011:941395

17. Takeda H, Nishikawa H, Iguchi E, Matsuda F, Kita R, Kimura T, et al. Sorafenib-induced acute interstitial pneumonia in patients with advanced hepatocellular carcinoma: report of three cases. Clin J Gastroenterol. 2012; 5(4):407-12. 